UNITE MIXTE DE RECHERCHE

EN ECONOMIE PUBLIQUE

JOINT RESEARCH UNIT IN PUBLIC ECONOMIC

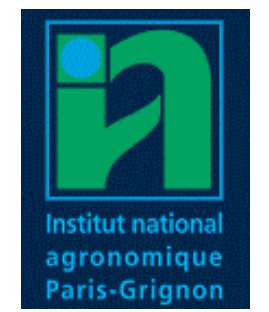

2006

CAHIERS DE RECHERCHE

WORKING PAPERS

2006/03

The Global Warming Potential Paradox:

Implications for the Design of Climate Policy

S. De Cara, E. Debove, P.A. Jayet

UMR Economie Publique

BP 01 - 78850 Grignon

16 rue Cl. Bernard - 75005 Paris

Tel. +33(0)130815330

Fax. +33 (0)130815368

http://www.grignon.inra.fr/economie-publique 


\title{
The Global Warming Potential Paradox: Implications for the Design of Climate Policy*
}

\author{
Stéphane De Cara \\ Institut National de la Recherche Agronomique (INRA), UMR Economie Publique INRA INA-PG, \\ Grignon, France and International Institute for Applied Systems Analysis (IIASA), Laxenburg, \\ Austria \\ Elodie Debove \\ Institut National de la Recherche Agronomique (INRA), UMR Economie Publique INRA INA-PG, \\ Grignon, France \\ Pierre-Alain Jayet \\ Institut National de la Recherche Agronomique (INRA), UMR Economie Publique INRA INA-PG, \\ Grignon, France
}

This version: March 9, 2006

Running title: The GWP Paradox and the design of climate policy

\footnotetext{
* This was article was written while Stéphane De Cara was a Guest Research Scholar with the Forestry Program at IIASA. The hospitality of the Institute is gratefully acknowledged. The authors would like to thank Brian O'Neil, Michael Obersteiner, Eric Nævdal, and Richard Tol for helpful discussions. Thanks are also due to participants at EAERE Conference (Bremen, 2005) and CESifo Venice Summer Institute (David Bradford Memorial Conference: "The Design of Climate Policy", 2005). The usual disclaimer applies.
} 
The Global Warming Potential Paradox: Implications for the Design of Climate Policy

\begin{abstract}
We address the issue of the design of climate policy in a dynamic, multi-greenhouse gas context. Despite well-established shortcomings, the Global Warming Potential (GWP) is the most commonly used index to compare greenhouse gases. We first review the shortcomings of the GWP from an economic perspective and examine some of the possible reasons for its success in the climate negotiations so far. We then examine the analytical properties of a second-best GWP-based emission target and compare the resulting second-best abatement paths with the first-best ones. We particularly show that the second-best $\mathrm{CO}_{2}$-equivalent target must exceed the $\mathrm{CO}_{2}$ equivalence of first-best abatements in order to reduce the bias induced by the GWP.
\end{abstract}

Keywords: Global Warming Potential; climate change; climate policy; multigreenhouse gas agreements.

JEL codes: Q25. 


\section{Le Paradoxe du Pouvoir Radiatif Global: Implications pour la Politique}

Climatique

Résumé. Nous examinons la question de la définition de la politique climatique dans un contexte dynamique et multi-gaz. Malgré les difficultés d'ordre conceptuel qu'il soulève, le PRG est la métrique la plus communément utilisée pour comparer les gaz à effet de serre. Nous passons tout d'abord en revue les implications et les limitations de ce concept du point de vue de l'analyse économique, et analysons quelques-unes des raisons qui ont fait malgré tout son succès dans les négociations climatiques. Nous étudions ensuite les propriétés analytiques d'une cible de second rang fondée sur l'utilisation du PRG, et comparons la trajectoire des réductions d'émissions qui en résulte avec la trajectoire optimale. Nous montrons en particulier que l'abattement total (exprimé en équivalents $\mathrm{CO}_{2}$ ) doit être plus important avec une cible de second rang fondée sur le PRG qu'en régime de premier rang.

Mots-clés: Pouvoir Radiatif Global; changement climatique; politique climatique; accords multi-gaz.

Codes JEL: Q25. 


\section{Introduction}

Few concepts derived from natural sciences have made their way into international law. The Global Warming Potential, or GWP for short, is one of them. In its Article 5.3, the Kyoto Protocol states that "the global warming potentials used to calculate the carbon dioxide equivalence of anthropogenic emissions [...] of greenhouse gases [...] shall be those accepted by the Intergovernmental Panel on Climate Change" (UNFCCC, 1997). The wording of the Kyoto Protocol therefore passes on the "legally-binding" nature of the Kyoto emission targets to the GWP concept itself.

As a matter of fact, the success of the GWP in the international negotiation arena may be seen as a failed attempt by economists to have sound economics translated into policy instruments. As soon as the early nineties, while the GWP concept was gaining momentum in both the scientific and policy debates, some of the most prominent economists in the field of climate change questioned its use for greenhouse gas (GHG) comparison purposes (Eckhaus, 1992; Schmalensee, 1993; Reilly and Richards, 1993). The concept was attacked on the grounds that it misleads the economically-sound choice of the mitigation mix. In other words, the GWP sets a "wrong currency" for comparing various GHGs. These criticisms did not stem from a narrow-minded perspective on the issue, whereby any concept coming from outside of the economics paradigm would be ignored or discarded. Rather, scholars who made the case against the GWP were very much involved in interdisciplinary work undertaken at some of the world's top climate-change research institutions. A dozen years of research later, one is left with the conclusion that the fundamental economic message contained in those criticisms was not successfully conveyed. Hereafter, we refer to this failure as the GWP paradox. Bradford (2001) summarized the paradox as follows:

"In general, natural scientists have been attracted to the GWP concept because of its purely physical quality. Although economists have argued that the tradeoffs cannot be inferred from physical properties alone, but have an inherent 
economic and policy dimension in terms of targets, the message has been slow to be accepted in the scientific community."

The term "slow" in Bradford's analysis should be understood as a euphemism. The GWP has stood as a key feature in all assessment reports hitherto published by the IPCC (Houghton et al., 1990; 1995; 2001). Despite the caveats that were included in the latest IPCC Assessment Report (Ramaswamy et al., 2001), the importance of the GWP is not likely to fade away any time soon, and certainly not before the end of the first Kyoto commitment period. The concept is even commonly used by economists. Partly because of the status conferred by its inclusion in the Kyoto Protocol, and partly because the inherent economic inconsistencies it implies have been overlooked, the vast majority of economic assessments of the costs and/or the benefits of multi-gas mitigation strategies rely on the GWP concept.

Yet the result that the GWP is ill-defined is a robust one from an economic standpoint. It has been confirmed by a number of studies, which, following up on the aforementioned pioneering works, proposed alternative indices (Kandlikar, 1995; Kandlikar, 1996; Hammit et al., 1996; Bradford and Keller, 2000; Manne and Richels, 2001; Shine et al., 2005) or provided empirical assessments of the concept's implications (Michaelis, 1999; Smith and Wigley, 2000; O’Neil, 2000; Tol et al., 2003; O’Neil, 2003; Kurosawa, 2004; Sarofim et al., 2005). See Fuglestvedt et al. (2003) for a comprehensive review. Given the success of this "wrong currency", one is forced to admit that some aspects of the GWP must have been sufficiently compelling to outweigh the major shortcomings that critics have been consistently pointing out.

Two strategies may then be envisaged to address multi-greenhouse gas issues. The continuation of frontal attacks to the GWP is one. This would involve continually trying to fit important economic concepts such as discounting, marginal abatement costs and marginal damage into the definition of a GHG index in the hope that this will eventually prove more successful than it has been in the last decade. An alternative - and more modest - approach focuses on second-best GWP-based economic instruments. This alternative approach recognizes that the GWP, albeit imperfect, must have compelling aspects that made it so successful as a policy con- 
cept. The challenge thus consists in designing economic instruments able to minimize the bias induced by the use of an imperfect metric. This latter approach is the one explored in the present paper.

Flexibility is put forward as a key component of a successful, cost-effective climate policy architecture. Flexibility is commonly categorized into "where"-, "when"-, and "what"-flexibility. The debate over any GHG equivalence rule underlines the importance of GHG trade-offs and, therefore, is logically linked to the "what"-flexibility issue (Börhinger et al., 2005). The estimated cost-savings permitted by "what"flexibility are high, especially when one compares the costs associated to $\mathrm{CO}_{2}$-only strategies with that of multi-GHG mitigation strategies (Reilly et al., 1999; Hayhoe et al., 1999). The magnitude of expected cost-savings related to additional "what"-flexibility certainly played a role in the success of the GWP concept.

Implications of the issues that are raised by the GWP however go beyond "what"flexibility. They cannot be disconnected from the analysis of "where"- and "when"flexibility. First, at the core of the critical views of the GWP concept lies the tradeoff between short- and long-lived GHGs (Aaheim, 1999; Michaelowa, 2003). The definition of any equivalence rule between various GHGs is thus crucial for the timing of mitigation strategies. Second, the GWP debate is also strongly linked to "where"flexibility in both a sectoral and geographical sense. The relative contribution of non$\mathrm{CO}_{2}$ emissions varies widely across sectors and countries. In this regard, agriculture provides a good illustration. The contribution of this sector, which is the major emitting sector for non- $\mathrm{CO}_{2}$ GHGs, to global reductions in GHG emissions heavily depends on the value attached to methane and nitrous oxide abatements relatively to $\mathrm{CO}_{2}$ abatements. Given the importance of agriculture in developing countries' economies and the high share of non- $\mathrm{CO}_{2}$ emissions from rice cultivation (methane), nitrogen fertilization (nitrous oxide), and livestock production (methane and nitrous oxide), multi-gas targets and the relative weights attached to non- $\mathrm{CO}_{2}$ gases may play a crucial role in getting developing countries on board in a post-Kyoto world. The stakes are thus high with regard to the design of a multi-gas climate policy architecture that is both negotiable and economically-sound. 
The paper is structured as follows. Section 1 reviews the shortcomings of the GWP from an economic perspective and investigates some possible reasons for its success in the design of climate policy to date. In Section 2, we present the framework used to address multi-greenhouse issues and derive the analytical properties of optimal multi-GHG abatement paths. The bias induced by the use of GWP-based targets is illustrated in Section 3. Section 4 discusses the properties of second-best GWPbased multi-GHG targets. Section 5 examines the policy and economic implications of GWP-based instruments.

\section{The GWP: Paradox or Pandora's Box?}

\subsection{The Shortcomings of the GWP From an ECONOMIC STANDPOINT}

As noted by Bradford, the GWP is of purely physical nature. It measures the timeintegrated radiative forcing caused by one emission pulse of one gas relatively to that of a reference gas - typically $\mathrm{CO}_{2}$ - over a given time horizon - typically 100 years (Ramaswamy et al., 2001).

Prior to further analyzing the paradoxical nature of the GWP success, let us first recall the formula that is explicitly referred to in the Kyoto Protocol (Ramaswamy et al., 2001, p. 385):

$$
\operatorname{GWP}_{j, \mathrm{CO}_{2}}(\hat{T})=\frac{\int_{0}^{\hat{T}} f_{j}(t) \cdot \theta_{j}(t) d t}{\int_{0}^{\hat{T}} f_{\mathrm{CO}_{2}}(t) \cdot \theta_{\mathrm{CO}_{2}}(t) d t}
$$

where $f_{j}(t)$ represents the time-dependent decay in abundance of the instantaneous release of gas $j$ at time $t=0 ; \theta_{j}(t)$ is the instantaneous radiative efficiency due to a unit increase in atmospheric abundance of gas $j$; and $\mathrm{CO}_{2}$ is taken as the reference gas.

All is fine as long the use of the GWP is restricted to synthetic reporting of the aggregate radiative impact caused by various GHGs. Such an index may well help in identifying and prioritizing mitigation options. But there is a conceptual leap between this use of the GWP and the interpretation of the GWP as an indicator of GHG relative prices. This leap was taken in Article 5.3 of the Kyoto Protocol. A di- 
rect consequence of the Kyoto multi-gas targets expressed in tons of $\mathrm{CO}_{2}$-equivalent and based on the use of GWP, was to set - at least in practice if not explicitly - the relative monetary value of abatements in methane, nitrous oxide, and other GHGs relatively to that of $\mathrm{CO}_{2}$. A project that entails an emission reduction of one ton of methane is thus entitled 21 times $^{1}$ as many "credits" as one project that entails an emission reduction of one ton of $\mathrm{CO}_{2}$. As soon as the GWP broke into the economic sphere by playing the role relative prices should play, it became an easy target for economists.

First, as indicated in equation (1), the GWP relies on the use of a finite time horizon, $\hat{T}$, which is the same for all GHGs. Strikingly, despite the sophisticated modeling effort required to describe radiative efficiency and atmospheric behavior of the various GHGs, one is left with a somewhat arbitrary choice when it comes to set $\hat{T}$. A 100-year time horizon is the convention taken in the national emissions inventories, although the IPCC also reports 20- and 500-year GWPs. As a matter of fact, scientific arguments supporting the use of 100-year instead of any other time horizon are lacking. Indeed, the index is quite sensitive to the chosen time horizon.

Second, the treatment of time implied by the use of the GWP is questionable. Indeed, by using a cut-off time, $\hat{T}$, and no discounting, the GWP concept substantially diverges from the traditional economic treatment of time. Although discounting is one of the most debated issues in economics (Weitzman, 1998), it is widely accepted that comparison of costs and revenues occurring at various points in time should be based on a consistent measure of welfare. This requires accounting for the possibility of inter-temporal arbitrage (e.g., through saving and investment), for future growth, and for agents' impatience and risk aversion when comparing a change in today's consumption with a change in future consumption. If examined through the lenses of the discounting theory, the GWP implies a discount rate that is flat and equal to zero within the next hundred years (if $\hat{T}=100$ ), and jumps to infinity for the subsequent future (Reilly et al., 2001). This would hardly be supported by economic theory (see also Fearnside, 2002;Tol, 2002a). As an illustration, the GWP would be the same, if the climate impact were felt in the first year or in the ninetieth year. 
By contrast, climate impacts occurring after one hundred years are totally ignored. Two abatement profiles involving different gas mixes may well be equivalent in terms of $\mathrm{CO}_{2}$-equivalent, but significantly differ in terms of climate and welfare impacts (Fuglestvedt et al., 2000).

Third, the GWP implies equivalence factors that are constant over time since it is based on today's atmospheric compositions in each GHG and radiative forcing. Given the dynamic nature of climate and atmospheric responses, today's GWP is thus likely to provide an inaccurate index for comparing future emissions of GHGs.

The fourth argument used against the GWP is more fundamental and encompasses the three aforementioned criticisms. In a market-based economy, the price of any good is the reflection of the value attached to this particular good relative to that of a numeraire. Relative prices depend on the scarcity of goods measured against that of the numeraire, which is itself driven by preferences and costs. Relative prices are thus intrinsically linked to a measure of welfare. Using the GWP as the price of any GHG relatively to $\mathrm{CO}_{2}$ therefore implies a confusion between impact - as calculated in the GWP, expressed in terms of time-integrated radiative forcing - and damage. In short, what matters in the economic analysis of climate-change is not climate change per se, but how it affects welfare in the broadest sense. The latter can be monetized, at least to a certain extent, for instance through measuring the loss in consumption due to the occurrence of climate change. In order to act as a relative price, the GWP thus does not target the right variable - and therefore sends a wrong signal—, unless one assumes impact and damage are linearly linked (Kandlikar, 1996). Assuming such a linear link is equivalent to assuming that multiplying radiative forcing by two (or ten, or one hundred) would make agents two (or ten, or one hundred) times worse off. Rather, evidence suggests that both climate and human systems responses are characterized by strong non-linearities.

In addition, the GWP is of little use, if at all, for atmospheric and climate models, which generally deal with each GHG separately. The GWP is thus merely restricted to reporting purposes in natural sciences. 
1.2. Why Did The GWP SUCCEED, WhILE ECONOMIC INDICES FAILED?

Alone, any of the criticisms discussed above could have been sufficient to downplay the importance of the GWP, and favor the use of better-suited, alternative GHG indices. The puzzling success story of the GWP is better understood if the GWP is viewed as a policy concept, not a scientific one. And as for many policy concepts, its development resulted from compromises, which were perceived as utterly necessary for policy steps to be taken, be it at the expense of economic soundness.

The importance of non- $\mathrm{CO}_{2}$ gases from a cost-effectiveness perspective was a key driver for the development of a GHG index. In the early development stages of the climate issue, research was focused almost exclusively on $\mathrm{CO}_{2}$ emissions ${ }^{2}$, not only because of the prime role of $\mathrm{CO}_{2}$ in the enhanced greenhouse effect phenomenon, but also because of its straightforward link with fossil fuel use, and therefore with economic growth. At the time the Kyoto targets were being negotiated, it became clear that the restriction of mitigation efforts solely to the energy and transport sectors would lead to very high abatement costs. Other mitigation options were thus needed in order to lower the overall abatement burden. Expanding the "basket of gases" to include non- $\mathrm{CO}_{2}$ gases in the agreement was one means of putting forward significant reduction targets that would not have been possible otherwise. As shown by De Cara and Rotillon (2003), multi-gas targets are also determinant in reaching a broader stable agreement. For a variety of reasons, the GWP was perceived as a scientifically documented concept that could be fairly easily explained to the public and accepted by the negotiating parties. At the time the Kyoto Protocol was drafted, this proved helpful in setting multi-gas, rather than $\mathrm{CO}_{2}$-only, targets.

Alternative indices proposed in the economic literature pursued the same objective, but did not encounter the same success. The promoters of an economicallysound index posed the right questions. But, as we argue hereafter, they fell short of delivering timely answers that could have been perceived as robust enough to be used in practice. Let us examine what was in the "Pandora's box" that economists dared to open. 
First, the amount of information required to compute these indices is much greater than for the GWP. In a cost-benefit framework, the welfare-based index of gas $j$ relative to gas $k$ is defined by the ratio of the shadow price of gas $j$ over that of gas $k$. The shadow price of gas $j$ reflects the present value of the flow of marginal damage due to an emission of gas $j$. Along an optimal trajectory, it should be equal to the marginal abatement cost of gas $j$. Computing such an index is admittedly challenging. In addition to the knowledge of climate and atmospheric responses - also needed to calculate GWPs - this requires estimating the flow of damage and abatement costs. Even in a more modest cost-effectiveness approach, whereby one seeks the least-cost abatement trajectory in order to meet some predefined concentration or temperature change target, abatement costs for all GHGs need to be estimated and extrapolated for the whole planning horizon. As most of the modelling effort was oriented toward $\mathrm{CO}_{2}$ mitigation in the energy sector, only little was known about non- $\mathrm{CO}_{2}$ abatement costs.

Second, uncertainties are compounded at all stages of the causality chain that proceeds from emissions to the translation of climate change impacts into economic terms. An economic GHG index inherently encompasses all the links in this causality chain. As a result, the foundations of such an index were vulnerable to strategic manipulations by the parties. The same argument applies to the introduction of discounting in the index. Using a discounted welfare-based index forces to explicitly state how the long-run is valued against the short-run. By how much, or if at all, future costs and benefits should be discounted is still very much debated among economists. Arguably, reaching an agreement among Kyoto negotiators on a common explicit discount rate would have been challenging.

Third, climate models generally agree on the fact that impacts will not be uniformly distributed across regions. Nor will be economic losses. Directional "exchange rates"- such as those analyzed by Førsund and Nædval (1998) in the sulfur emission problem - provide an interesting analogy in this respect. A GHG index should then reflect the fact that damages will vary not only in time, but also in space. In concrete terms, this would involve a country- or at least region-differentiated GHG index. 
Conceivably, given the uncertainties affecting regional damage estimates, this might again have opened the way to strategic manipulations and undermined the likelihood of reaching an agreement on multi-gas targets.

That is not to say that the GWP provides better answers to any of these fundamental difficulties. Instead, as discussed above, the GWP - wrapped in an aura of "pure" natural science - simply avoids them and implicitly makes simplistic assumptions with regard to preferences, damage, and discounting. Should these assumptions have come from economics, they would not have passed the test.

Where do we stand? First, with the balance in economic research about climate change shifting from energy-only to agriculture and land-use issues, non- $\mathrm{CO}_{2}$ emission sources and abatement costs are now better understood (Hayhoe et al., 2000; McCarl and Schneider, 2001; Reilly et al., 2003; De Cara et al., 2005). Second, more results are now available on the assessment of climate change economic costs, including spatially differentiated ones, and this field is still being intensively researched (Mendelsohn et al., 1998; Tol, 2002b). All uncertainties are far from being resolved, but important progress has been made in quantifying these uncertainties (Tol, 2003). Unfortunately, the results from this research have come too late to play their due part in the Kyoto Protocol. Now that it is carved in the stone of the Kyoto Protocol, the GWP has to be dealt with by economists. However, the latest economic results can be helpful in designing instruments aimed at fixing (at least some of) the flaws of the GWP concept.

\section{Optimal multi-gas abatement path}

In this section, we develop an analytical framework to investigate multi-greenhouse gas issues. The analytical properties of the first-best solution are first examined in order to use them later as a benchmark in the analysis of GWP-based instruments.

Consider a problem with $n$ greenhouse gases indexed by $j=1, \ldots, n$. The $n$ vector $^{3}$ of atmospheric concentrations at time $t$ is denoted by ${ }^{t} \mathbf{z}_{t}=\left(z_{1 t}, \ldots, z_{n t}\right)$. GHGs tend to decay in the atmosphere. Most of the literature that examines multi- 
gas issues from an analytical perspective assumes exponential decay processes characterized by constant decay rates (Moslener and Requate, 2005, for instance). This assumption has the advantage of greatly simplifying the computation of the optimal control problem by restricting it to a linear differential system. However, it overlooks two important features of the atmospheric behavior of GHGs. First, because of the complexity of the exchanges between different carbon reservoirs (atmosphere, ocean, terrestrial carbon pool) characterized by different transfer speeds between each of them, the carbon cycle can hardly be reduced to a simple, constant-rate decay process (Houghton et al., 2001). Second, interactions between the various GHGs in the atmosphere can significantly impact the speed at which they are decayed (Houghton et al., 2001). Therefore, we adopt here a fairly general formulation of the decay process. We represent this process by the $n$-vector valued function $\mathbf{f}\left(\mathbf{z}_{t}\right)={ }^{t}\left(f_{1}\left(\mathbf{z}_{t}\right), \ldots, f_{n}\left(\mathbf{z}_{t}\right)\right)$. Each entry of $\mathbf{f}()$, denoted by $f_{j}(\mathbf{z})$, describes the decay process of gas $j$ as a function of the full vector of concentrations.

Anthropogenic emissions in all GHGs are denoted by the $n$-vector ${ }^{t} \mathbf{e}=\left(e_{1 t}, \ldots, e_{n t}\right)$ and are measured in mass unit of each gas (tons of $\mathrm{CO}_{2}$, tons of methane, etc.). Net emissions are decomposed into two components: (i) business-as-usual emissions, which are denoted by ${ }^{t} \overline{\mathbf{e}}_{t}=\left(\bar{e}_{1 t}, \ldots, \bar{e}_{n t}\right)$, and (ii) abatements, which are denoted by ${ }^{t} \overline{\mathbf{a}}_{t}=\left(a_{1 t}, \ldots, a_{n t}\right)$. The business-as-usual emission path is considered exogenous, and can be taken for instance from the IPCC scenarios (Nakicenovic and Swart, 2000).

The equation of motion over time of concentration is thus:

$$
\dot{z}_{j t}=-f_{j}\left(z_{1 t}, \ldots, z_{n t}\right)+\bar{e}_{j t}-a_{j t} \quad \text { for } j=1, \ldots, n
$$

or, in matrix form:

$$
\dot{\mathbf{z}}_{t}=-\mathbf{f}\left(\mathbf{z}_{t}\right)+\overline{\mathbf{e}}_{t}-\mathbf{a}_{t}
$$

The effect of GHGs on the climate is measured by $\theta\left(\mathbf{z}_{t}\right)$, which summarizes climate change through, for instance, the change in global mean surface temperature. $\theta($. depends on the full vector of concentrations, accommodating the possible countereffects that some gases can exert on the radiative impact of other gases. This is 
particularly important to account for the interactions between the radiative forcing of methane and that of nitrous oxide in the atmosphere (Ramaswamy et al., 2001).

In a welfare-based analysis of multi-GHG issues, climate impacts need to be translated into damages. Following a commonly used assumption in the literature, we consider that the economic loss due to climate change, denoted by $D\left(\theta\left(\mathbf{z}_{t}\right)\right)$, is an increasing and convex function of the change in global surface temperature $(D(0)=$ $\left.0, D^{\prime}()>0,. D^{\prime \prime}() \geq 0.\right)$. As we consider the optimal emission path from a global perspective, we focus on the global measure of the damage, not on differentiated damages (see discussion above).

Abatement costs at time $t$, denoted by $C\left(\mathbf{a}_{t}\right)$, depend on the level of abatements in all GHGs. Again, a general formulation is important to account for potential interactions between the processes governing emissions, as well as between mitigation strategies. Agriculture provides an interesting illustration of such interactions. Mitigation strategies with respect to enteric fermentation (mostly methane) can take the form of reducing livestock numbers and/or modifying the way animals are fed. Both options have impacts on emissions from manure management (methane, but also nitrous oxide) and emissions from agricultural soils (mostly nitrous oxide). Separability of abatement costs - an assumption often retained in the literaturebetween methane and nitrous oxide is thus hardly justified. In the general framework developed in this paper, we account for these interactions. However, for the sake of simplicity, we retain a quadratic ${ }^{4}$ formulation:

$$
C\left(a_{1 t}, \ldots, a_{n t}\right)=\frac{1}{2} \sum_{j=1}^{n} \sum_{k=1}^{n} c_{j k} a_{j t} a_{k t}
$$

or, in matrix form:

$$
C\left(\mathbf{a}_{t}\right)=\frac{1}{2}{ }^{t} \mathbf{a}_{t} \cdot \mathbf{C} \cdot \mathbf{a}_{t}
$$

That is, each entry of the vector of marginal abatement costs in all GHGs is assumed to be linear with respect to the vector of abatements $(\mathbf{C} \cdot \mathbf{a})$. In order to fulfill the usual convexity requirements, $\mathbf{C}$ is a $n \times n$, symmetric, and positive definite matrix. See Moslener and Requate (2005) for a discussion of the importance of non-diagonal entries in matrix $\mathbf{C}$. In addition, note that equation (5) implies that 
the abatement cost function is constant over time. That is, the formulation does not account for technical progress in the abatement technology, neither through an exogenous cost-decreasing trend nor a learning-by-doing process. Although this can arguably be important in deriving optimal targets, this would increase the complexity of the subsequent developments without fundamentally changing the nature of the results.

We now turn to the problem faced by a (risk-neutral) social planner, who seeks to set optimal abatement trajectories in all gases in order to minimize the sum of abatement costs and damage. $\rho$ denotes the (constant) social discount rate. The corresponding program is:

$$
\min _{\mathbf{a}_{t}} \int_{0}^{+\infty}\left[C\left(\mathbf{a}_{t}\right)+D\left(\theta\left(\mathbf{z}_{t}\right)\right)\right] e^{-\rho t} d t \text { subject to }(3) \text { and } \mathbf{a}_{t} \leq \overline{\mathbf{e}}_{t}
$$

The inequality constraint in program (6) reflects the fact that abatements cannot exceed business-as-usual emissions. In fact, whether this constraint is binding at any point in time depends on the business-as-usual emission path and on the shape of abatement costs. In addition, one may argue that for some gases (e.g. $\mathrm{CO}_{2}$ ), some mitigation options might allow, at least in theory, to abate more than what is emitted (e.g. carbon sequestration). Therefore, we assume that abatement costs are such that they prevent abatement to exceed business-as-usual emissions and focus on interior solutions.

The first-order optimality conditions for program (6) are given by ${ }^{5}$ :

$$
\begin{aligned}
\mathbf{a}_{t}^{*} \in \arg \min _{\mathbf{a}_{t}} \mathcal{H}^{C} & =C\left(\mathbf{a}_{t}\right)+D\left(\theta\left(\mathbf{z}_{t}^{*}\right)\right)+{ }^{t} \boldsymbol{\mu}_{t}^{*} \cdot\left(-\mathbf{f}\left(\mathbf{z}_{t}^{*}\right)+\overline{\mathbf{e}}_{t}-\mathbf{a}_{t}\right) \\
\dot{\mu}_{j t}^{*} & =\rho \mu_{j t}^{*}-\frac{\partial \mathcal{H}^{C}}{\partial z_{j}} \text { for all } j=1, \ldots, n
\end{aligned}
$$

where $\mathcal{H}^{C}$ denotes the current Hamiltonian of program (6), $\boldsymbol{\mu}_{t}$ denotes the $n$-vector of adjoint variables (or shadow prices) associated with the $n$ equations of motion. Optimal levels of state, control and adjoint variables are denoted with a star.

The relationships (7a)-(7b), together with the equation of motion of $\mathbf{z}_{t}(3)$, initial concentrations $\mathbf{z}_{0}$, and transversality conditions characterize the optimal abatement trajectories. 
Equation (7a) implies that, at all point in time, optimal abatement in all gases should be such that marginal abatement in gas $j$ is equal to the respective shadow price, $\mu_{j t}^{*}$. From equation (5), we can derive the optimal abatement supply in all gases as a function of the vector of shadow prices:

$$
\mathbf{a}_{t}^{*}=\mathbf{C}^{-1} \cdot \boldsymbol{\mu}_{t}^{*}
$$

Equations $(7 \mathrm{~b})$ can be rewritten in matrix form as:

$$
\dot{\boldsymbol{\mu}}_{t}^{*}=\left(\rho \mathbf{I}_{n}+\mathbf{J}_{\mathbf{f}}\left(\mathbf{z}_{t}^{*}\right)\right) \cdot \boldsymbol{\mu}_{t}^{*}-D^{\prime}\left(\theta\left(\mathbf{z}_{t}^{*}\right)\right) \mathbf{J}_{\theta}\left(\mathbf{z}_{t}^{*}\right)
$$

where $\mathbf{I}_{n}$ is the $n \times n$-identity matrix, $\mathbf{J}_{\mathbf{f}}\left(\mathbf{z}_{t}\right)$ is the $n \times n$-Jacobian matrix of $\mathbf{f}\left(\mathbf{z}_{t}\right)$, whose generic entry is defined as $\frac{\partial f_{j}}{\partial z_{k}}\left(\mathbf{z}_{t}\right)$, and $\mathbf{J}_{\theta}\left(\mathbf{z}_{t}\right)$ is the $n \times 1$-Jacobian matrix of $\theta\left(\mathbf{z}_{t}\right)$, whose generic entry is $\frac{\partial \theta}{\partial z_{j}}\left(\mathbf{z}_{t}\right)$. The $j$-th row of the matrix $\mathbf{J}_{\mathbf{f}}\left(\mathbf{z}_{t}\right)$ is thus the profile of the marginal impact of a change in the atmospheric composition on gas $j$ 's concentration. Similarly, the $j$-th entry of $\mathbf{J}_{\theta}\left(\mathbf{z}_{t}\right)$ reflects the marginal impact of gas $j$ on global temperature.

Equation (9) imposes that each individual shadow price changes over time in such a way that it equals the present value of damage caused by a marginal increase in emissions in the respective gas. Introducing optimal abatements from equation (8) into equation (3) yields:

$$
\dot{\mathbf{z}}_{t}^{*}=-\mathbf{f}\left(\mathbf{z}_{t}^{*}\right)+\overline{\mathbf{e}}_{t}-\mathbf{C}^{-1} \boldsymbol{\mu}_{t}^{*}
$$

Together, equations (9) and (10) define a $2 n$ first-order differential system in $\boldsymbol{\mu}_{t}$ and $\mathbf{z}_{t}$. Note that if $D($.$) is linear with respect to \theta$, and $\mathbf{f}($.$) and \theta($.$) are both linear$ with respect to $\mathbf{z}_{t}$, then equation (9) reduces to a linear first-order differential system with constant coefficients. The sub-system in $\boldsymbol{\mu}_{t}$ can then be solved independently of $\mathbf{z}_{t}$. In this case, the full system can be solved stepwise, solving first for $\boldsymbol{\mu}_{t}$, then computing optimal abatement through equation (8), and finally solving for concentrations through equation (10). As soon as the non-linearities in either damage, climate responses, or atmospheric concentrations are taken into account, this simple step-wise solving method does not apply. Given our fairly general assumptions with respect to these variables, solving the full system is more of a numerical task than an analytical one. 


\section{GWP-based abatement targets}

Optimal responses to multi-dimensional issues generally require as many instruments as there are dimensions in the problem. Multi-GHG issues are not different. The previous section examined the solution of a $n$-dimensional problem ( $n$ GHGs) for which the social planner has $n$ command variables at hand ( $n$ abatement paths). The use of the GWP, or of any constant metric in that regard, leaves the social planner with only one command variable by summarizing the full profile of emissions into one scalar (e.g., total $\mathrm{CO}_{2}$-equivalent abatement). Yet, the problem is still a $n$ dimensional one as soon as GHGs atmospheric behavior and climate impacts differ. By construction, GWP-based instruments are therefore likely to provide sub-optimal answers to multi-GHG issues. In this section, we examine the implications of GWPbased abatement targets.

Consider that a GWP-like GHG index has been agreed upon. This index allows converting emissions in any gas $j$ into one particular reference gas. Without loss of generality, we assume that gas 1 is taken as the reference gas. For clarity of the exposition and in order to stay in line with the Kyoto Protocol's terminology, we refer to gas 1 as $\mathrm{CO}_{2}$. Let ${ }^{t} \boldsymbol{\gamma}=\left(1, \gamma_{2}, \ldots, \gamma_{n}\right)$ be the $n$-vector of conversion coefficients of gas $j$ into $\mathrm{CO}_{2}$. As is the case for the GWPs, all entries of $\gamma$ are assumed to be constant over time. Note that $\gamma$ encompasses the standard definition of the GWP as a particular case, but also covers any kind of constant GHG index. As an illustration, $\mathrm{CO}_{2}$-only strategies can also be analyzed using this framework (in this case, $\gamma_{j}=0$ for all $j \geq 2$ ). Total $\mathrm{CO}_{2}$-equivalent abatement at time $t$ amounts to:

$$
\sum_{j=1}^{n} \gamma_{j} a_{j t}={ }^{t} \boldsymbol{\gamma} \cdot \mathbf{a}_{t}
$$

We proceed in two steps. First, we assume that a $\mathrm{CO}_{2}$-equivalent target for the entire planning horizon has been set by the social planner. Agents adjust their abatement decisions in order to minimize the cost of meeting this target at all times. Second, the social planner chooses the optimal target knowing agents' responses. 
Let us thus first assume that an aggregate target, $\alpha_{t}$, based on the $\boldsymbol{\gamma}$-index, has been fixed. The corresponding cost minimization program is:

$$
\min _{\mathbf{a}_{t}} C\left(\mathbf{a}_{t}\right) \text { subject to }{ }^{t} \boldsymbol{\gamma} \cdot \mathbf{a}_{t} \geq \alpha_{t}
$$

which leads to the $n$ following first-order conditions written in matrix form:

$$
\mathbf{C} \cdot \mathbf{a}_{t}=\lambda_{t} \gamma
$$

The abatement profile at time $t$ should be such that the marginal abatement cost in each gas is equal to the shadow price associated with the $\gamma$-aggregated target $\left(\lambda_{t}\right)$ times the respective value of the GHG index. Note that at the optimum $\lambda_{t}$ should thus be equal to the marginal abatement cost of $\mathrm{CO}_{2}$. If abatements are to be traded through a single emission permit system, the equilibrium price of gas $j$ on this market should be equal to $\gamma_{j} \lambda_{t}$. This illustrates the fact that a multi-gas target sets the relative prices of the various GHGs.

By using the fact that the constraint in program (12) should be binding at the optimum, we can eliminate $\lambda_{t}$. The cost-minimizing abatement vector is denoted by a tilde and is obtained as a function of the $\mathrm{CO}_{2}$-equivalent target and $\gamma$ :

$$
\tilde{\mathbf{a}}\left(\alpha_{t}\right)=\frac{\alpha_{t}}{{ }^{t} \boldsymbol{\gamma} \mathbf{C}^{-1} \boldsymbol{\gamma}} \mathbf{C}^{-1} \boldsymbol{\gamma}
$$

Imagine now that the social planner uses the first-best abatement profile, $\mathbf{a}_{t}^{*}$, to compute $\alpha_{t}$. That is, $\alpha_{t}$ is chosen to be equal to $\alpha_{t}^{*}={ }^{t} \boldsymbol{\gamma} \cdot \mathbf{a}^{*}$, with $\mathbf{a}_{t}^{*}$ defined as in equation (8). Admittedly, one may find it odd that GWP-based targets are to be used whereas first-best abatements are assumed to be known. This indeed exemplifies the GWP paradox discussed in Section 1. Using equations (8) and (14), this yields:

$$
\tilde{\mathbf{a}}\left(\alpha_{t}^{*}\right)=\frac{{ }^{t} \gamma \mathbf{C}^{-1} \boldsymbol{\mu}_{t}^{*}}{{ }^{t} \boldsymbol{\gamma} \mathbf{C}^{-1} \boldsymbol{\gamma}} \mathbf{C}^{-1} \boldsymbol{\gamma}
$$

Comparing this to the first-best vector of abatements yields:

$$
\tilde{\mathbf{a}}\left(\alpha_{t}^{*}\right)-\mathbf{a}_{t}^{*}=\mathbf{C}^{-1}\left(\frac{{ }^{t} \boldsymbol{\gamma} \mathbf{C}^{-1} \boldsymbol{\mu}_{t}^{*}}{{ }^{t} \boldsymbol{\gamma} \mathbf{C}^{-1} \boldsymbol{\gamma}} \boldsymbol{\gamma}-\boldsymbol{\mu}_{t}^{*}\right)
$$

Given our assumptions on $\mathbf{C}$, the only solution for the full profile of abatement $\tilde{\mathbf{a}}\left(\alpha_{t}^{*}\right)$ to be equal to the first-best full profile of abatements is such that $\boldsymbol{\gamma}=k \boldsymbol{\mu}_{t}^{*}$ 
where $k$ is any positive real scalar. Given the additional convention that $\gamma_{1}=1$, the only solution for $\tilde{\mathbf{a}}\left(\alpha_{t}^{*}\right)=\mathbf{a}_{t}^{*}$ to hold is that $\gamma_{j}=\frac{\mu_{j t}^{*}}{\mu_{1 t}^{*}}$ for all $j=1, \ldots, n$, and at all time. This result embeds the essence of the critical views of the GWP concept and illustrates the fundamental economic result with regard to the GHG equivalence factor. The (first-best) equivalence rule should be based on the shadow prices ratios.

Multi-GHG targets that are derived from the conversion of first-best abatements into $\mathrm{CO}_{2}$-equivalent result in a distortion of the abatement efforts. When valued at respective shadow prices, the resulting change in welfare is worth:

$$
{ }^{t} \boldsymbol{\mu}_{t}^{*} \cdot\left(\tilde{\mathbf{a}}\left(\alpha_{t}^{*}\right)-\mathbf{a}_{t}^{*}\right)=\frac{\left({ }^{t} \boldsymbol{\gamma} \mathbf{C}^{-1} \boldsymbol{\mu}_{t}^{*}\right)^{2}}{{ }^{t} \boldsymbol{\gamma} \mathbf{C}^{-1} \boldsymbol{\gamma}}-{ }^{t} \boldsymbol{\mu}_{t}^{*} \mathbf{C}^{-1} \boldsymbol{\mu}_{t}^{*}
$$

Since $\mathbf{C}$ is symmetric and positive definite, we know as a direct application of the Cauchy-Schwarz theorem that the right-hand side of (17) is negative, and equal to zero if and only if $\gamma_{j}=\frac{\mu_{j t}^{*}}{\mu_{1 t}^{*}}$ for all $j=1, \ldots, n$ and all $t$.

\section{Second-best, GWP-based abatement targets}

The next step in our analysis consists of setting the best possible $\mathrm{CO}_{2}$-equivalent target from a social welfare point of view. We know that GWP-based quantity instruments lead to a distortion in the abatement mix. The question is then: Is it possible to reduce this distortion? This section examines a class of second-best GWP-based instruments. The corresponding social planner's problem is:

$$
\min _{\alpha_{t}} \int_{0}^{+\infty}\left[C\left(\mathbf{a}_{t}\right)+D\left(\theta\left(\mathbf{z}_{t}\right)\right)\right] e^{-\rho t} d t \text { subject to }(3) \text { and } \mathbf{a}_{t}=\tilde{\mathbf{a}}\left(\alpha_{t}\right)
$$

For any level of the $\mathrm{CO}_{2}$-equivalent target $\alpha_{t}$, abatements are supplied according to equation (14). Introducing (14) in the objective function and in the equation of motion of $\mathbf{z}_{t}$, one can form the current Hamiltonian $\hat{\mathcal{H}}^{C}$ and derive the following first-order optimality conditions (optimal values are signaled with a hat):

$$
\begin{aligned}
\hat{\alpha}_{t} \in \arg \min _{\alpha_{t}} \hat{\mathcal{H}}^{C} & =\frac{\alpha_{t}^{2}}{2^{t} \boldsymbol{\gamma} \mathbf{C}^{-1} \boldsymbol{\gamma}}+D\left(\theta\left(\hat{\mathbf{z}}_{t}\right)\right)+{ }^{t} \hat{\boldsymbol{\mu}}_{t}\left(\overline{\mathbf{e}}_{t}-\mathbf{f}\left(\hat{\mathbf{z}}_{t}\right)-\frac{\alpha_{t} \mathbf{C}^{-1} \boldsymbol{\gamma}}{{ }^{t} \boldsymbol{\gamma} \mathbf{C}^{-1} \boldsymbol{\gamma}}\right)(19 \mathrm{a}) \\
\dot{\hat{\boldsymbol{\mu}}}_{t} & =\left(\rho \mathbf{I}_{n}+\mathbf{J}_{\mathbf{f}}\left(\hat{\mathbf{z}}_{t}\right)\right) \cdot \hat{\boldsymbol{\mu}}_{t}-D^{\prime}\left(\theta\left(\hat{\mathbf{z}}_{t}\right)\right) \mathbf{J}_{\theta}\left(\hat{\mathbf{z}}_{t}\right)
\end{aligned}
$$


Solving problem (19a) for $\hat{\alpha}_{t}$ yields:

$$
\hat{\alpha}_{t}={ }^{t} \boldsymbol{\gamma} \mathbf{C}^{-1} \hat{\boldsymbol{\mu}}_{t}
$$

The optimal $\mathrm{CO}_{2}$-equivalent target thus depends on the full vector of shadow prices derived from program (18). From equation (19b), one sees that $\hat{\boldsymbol{\mu}}_{t}$ is different from $\boldsymbol{\mu}_{t}^{*}$ as soon as current concentrations $\hat{\mathbf{z}}_{t}$ differ from the first-best levels $\mathbf{z}_{t}^{*}$, that is, if either $D(),. \theta($.$) and/or \mathbf{f}($.$) is non-linear. Introducing the expression of \hat{\alpha}_{t}$ in equation (14) gives the second-best vector of abatements:

$$
\hat{\mathbf{a}}_{t}=\frac{{ }^{t} \boldsymbol{\gamma} \mathbf{C}^{-1} \hat{\boldsymbol{\mu}}_{t}}{{ }^{t} \boldsymbol{\gamma} \mathbf{C}^{-1} \boldsymbol{\gamma}} \mathbf{C}^{-1} \boldsymbol{\gamma}
$$

which leads to the following equation of motion for $\hat{\mathbf{z}}_{t}$ :

$$
\dot{\hat{\mathbf{z}}}_{t}=-\mathbf{f}\left(\hat{\mathbf{z}}_{t}\right)+\overline{\mathbf{e}}_{t}-\frac{{ }^{t} \boldsymbol{\gamma} \mathbf{C}^{-1} \hat{\boldsymbol{\mu}}_{t}}{{ }^{t} \boldsymbol{\gamma} \mathbf{C}^{-1} \boldsymbol{\gamma}} \mathbf{C}^{-1} \boldsymbol{\gamma}
$$

From (19a), we know that $\hat{\alpha}_{t}$ minimizes $\hat{\mathcal{H}}^{C}$. Hence, any abatement profile different from $\hat{\mathbf{a}}_{t}$ should then induce a net welfare loss when valued at current shadow prices $\hat{\boldsymbol{\mu}}_{t}$. Therefore, we have:

$$
{ }^{t} \hat{\boldsymbol{\mu}}_{t} \cdot\left(\hat{\mathbf{a}}_{t}-\tilde{\mathbf{a}}_{t}\left(\alpha_{t}^{*}\right)\right)=\left(\hat{\alpha}_{t}-\alpha_{t}^{*}\right)\left(\frac{\hat{\alpha}_{t}}{{ }^{t} \boldsymbol{\gamma} \mathbf{C}^{-1} \boldsymbol{\gamma}}\right) \geq 0
$$

The loss in welfare is proportional to the difference between the second-best, $\mathrm{CO}_{2^{-}}$ equivalent target $\left(\hat{\alpha}_{t}\right)$ and the first-best abatement expressed in $\mathrm{CO}_{2}$ equivalent $\left(\alpha_{t}^{*}\right)$. As $\hat{\alpha}_{t}$ is positive, the optimal $\mathrm{CO}_{2}$-equivalent target has to exceed $\alpha_{t}^{*}={ }^{t} \boldsymbol{\gamma} \cdot \mathbf{a}^{*}$. Comparing the abatement profile prescribed by the first-best program and $\hat{\mathbf{a}}_{t}$ yields:

$$
\hat{\mathbf{a}}_{t}-\mathbf{a}_{t}^{*}=\mathbf{C}^{-1}\left(\frac{{ }^{t} \boldsymbol{\gamma} \mathbf{C}^{-1} \hat{\boldsymbol{\mu}}_{t}}{{ }^{t} \boldsymbol{\gamma} \mathbf{C}^{-1} \boldsymbol{\gamma}}-\boldsymbol{\mu}_{t}^{*}\right)
$$

This yields to a change in social welfare compared to the first-best abatement profile (valued at $\boldsymbol{\mu}_{t}^{*}$ ):

$$
{ }^{t} \boldsymbol{\mu}_{t}^{*} \cdot\left(\hat{\mathbf{a}}_{t}-\mathbf{a}_{t}^{*}\right)=\frac{{ }^{t} \gamma \mathbf{C}^{-1} \hat{\boldsymbol{\mu}}_{t}}{{ }^{t} \boldsymbol{\gamma} \mathbf{C}^{-1} \boldsymbol{\gamma}} \boldsymbol{\gamma} \mathbf{C}^{-1} \boldsymbol{\mu}_{t}^{*}-{ }^{t} \boldsymbol{\mu}_{t}^{*} \mathbf{C}^{-1} \boldsymbol{\mu}_{t}^{*}
$$

By comparing equations (25) and (17) and noticing that $\hat{\alpha}_{t}$ is greater than ${ }^{t} \boldsymbol{\gamma} \cdot \mathbf{a}^{*}$, one can easily see that setting $\hat{\alpha}_{t}$ as a $\mathrm{CO}_{2}$-equivalent target induces a welfare loss lower than if the $\mathrm{CO}_{2}$-equivalence of the first-best abatement profile is used. 


\section{Discussion: Policy and economic implications}

Non- $\mathrm{CO}_{2}$ GHGs are important in several respects. In the short run, they are likely to play a key role in closing the gap between the Kyoto targets and rising $\mathrm{CO}_{2}$ emission trends. The fact that a large share of the Clean Development Mechanism projects registered by the $\mathrm{UNFCCC}^{6}$ concentrate on non- $\mathrm{CO}_{2}$ emissions provides a good illustration in this regard. In the longer run, they can contribute to emission targets that have a significant impact on climate change and, at the same time, broaden the set of participating countries in a post-Kyoto architecture.

It is now well established that the GWP distorts the relative prices of GHGs, leading to over-abatement in some gases and under-abatement in others. In a wellfunctioning market system, "wrong" relative prices tend to prompt market responses that counterbalance the discrepancy between social value and market prices. But these forces can only play a limited role in the climate change issue, as the demand for lower pressure on climate is very imperfectly captured by the policymaking and negotiating process. Any multi-GHG targets based on the GWP will inevitably result in a mis-allocation of abatements.

The multi-gas issue can theoretically be solved by either setting targets for each individual GHG, or alternatively, assigning each GHG a price that adequately reflects the marginal abatement cost and the flow of future marginal damage. In the former case, individual GHG trading systems could be established to achieve cost-efficiency. In the latter case, trading could be done in a single $\mathrm{CO}_{2}$-equivalent market, the exchange rates between GHGs being fixed. The choice between price and quantity instruments would then rely on the discussion of the uncertainty affecting damage and costs. In both cases, instruments (either targets or prices) have to be regularly updated to account for the changes in atmospheric concentrations, and therefore in damage.

If, for some reason, the GWP still remains a cornerstone of the future design of climate policy, economic instruments have to be adapted to account for the resulting bias. That means, as shown in the previous section, that GWP-based targets have 
to be more stringent than the $\mathrm{CO}_{2}$-equivalence of the abatement path prescribed by integrated assessment models. The very fact that policymakers are left with only one command variable to cope with a multi-dimensional issue requires overshooting in order to offset (some of) the bias induced by the GWP.

The difference between first-best and second-best targets, when both are expressed in terms of GWP-based $\mathrm{CO}_{2}$-equivalent, is shown as depending on two factors. First, this difference depends on how the equivalence factors reflect the shadow prices of each respective GHG. Section 1 lists several reasons why the GWP, by construction, is not likely to be the right candidate to fulfill this objective. Even if, by mere chance, the vector of GWPs provided a good proxy for the vectors of the shadow prices at some point in time, this would not hold over time because shadow prices are subject to change as concentrations and damage change.

Second, the difference between first- and second-best $\mathrm{CO}_{2}$-equivalent targets depends on the gap between shadow prices under first- and second-best regimes. This, in turn, depends on the difference between first- and second-best concentration, temperature, and damage paths. For short-run targets, which correspond to small changes in concentrations, linear approximation might be adequate. In this case, the difference between first- and second-best shadow prices remains small, and so is the difference between first- and second-best $\mathrm{CO}_{2}$-equivalent targets. But, if GWPs are to be used for a longer time period and/or the dynamics of the system (either in concentrations, temperature, or damage) is characterized by strong non-linearities, this gap will have to rise in order to accommodate the increasing discrepancy between first- and second-best shadow prices.

An important policy implication is that second-best GWP-based targets have to be updated on a regular basis in order to account for the change in concentrations and damage. To determine how often the targets should be revised, one has to weight the transaction costs associated with negotiating them and the welfare impacts.

Non- $\mathrm{CO}_{2}$ marginal abatement costs play a key role. The importance given to one particular gas cannot be reduced to its impact on climate but should also weigh the costs at which abatements can be supplied. As it is, the GWP does not reflect these 
differences. Nor do the relative prices in any GWP-based system. Nevertheless, as shown in the previous section, marginal abatement costs enter the calculation of second-best GWP-based emission targets.

Interestingly, the establishment of second-best GWP-based instruments would also force clarifying the assumptions taken with respect to discount rate and damage, as these two elements determine the calculation of the target. As discussed above, reaching an agreement on these assumptions is arguably challenging. However, economists could make the case that clear and consistent assumptions in this regard are in any respect better than the simplistic ones implied by the use of GWP.

\section{Concluding remarks}

The paces of policymaking and research are seldom synchronized. Often, scientists have to wait years or decades before they can see their concepts and results translated into policy. And sometimes policy moves ought to be made before science has had time to establish the necessary results. Global warming is one of the few examples of intense dialogue between interdisciplinary research and policymaking. The adoption of the GWP as the GHG "currency", however, stands out as a contrasting failure in this overall successful picture.

Is that to say that economists should throw in the towel in the event that the GWP is preferred over economic indices in the future design of climate policy? The analysis conducted in this paper shows that some room exists to adapt GWP-based targets in order to correct some of the distortions caused by the GWP. The role of economists would then be to emphasize that a $\mathrm{CO}_{2}$-equivalent target has to be more stringent than what would be necessary with the right "currency".

How much larger the second-best targets should be is very much an empirical question. Our analytical results show that this depends on the difference between the GWP and the shadow prices, the magnitude of marginal abatement costs, and the (non)-linearities in both economic and ecological systems. Further research is needed to assess this gap based on state-of-the-art integrated modelling approach. 


\section{Notes}

1 The GWP of methane as estimated in the 1995 IPCC Second Assessment Report. In its Third Assessment Report, the IPCC has revised methane's GWP upwards to 23 (Ramaswamy et al., 2001). However, the 1995 SAR GWPs are used for the verification of compliance with the Kyoto commitments.

${ }^{2}$ In the early nineties, it was widely accepted that $\mathrm{CO}_{2}$ was to be the primary, if not the only, target in any action taken to combat climate change: "Whatever type of international agreement is reached during the next decade, it will probably only cover $\mathrm{CO}_{2}$, not other climate gases. [...] Although agreements encompassing all climate gases could be more efficient, practical considerations, will thus force governments, at least initially, to limit an agreement to $\mathrm{CO}_{2} . "$ (Hoel, 1991). This prediction was proved wrong by later developments of the climate negotiations.

${ }^{3}$ Vectors and matrices are denoted in bold lower- and upper-case, respectively. All vectors are column vectors. The prescript ${ }^{t}$ denotes the transposed operator.

4 The fact that marginal abatement costs in each gas are linear with respect to the whole vector of abatements a greatly simplifies the calculation of the abatement supply (equation (8)). More general assumptions on the abatement cost function are possible, provided that the Hessian matrix of $C(\mathbf{a})$ is positive definite. This would require making use of the implicit function theorem and does not change the qualitative nature of the results.

${ }^{5}$ The convention taken here for the sign of shadow prices implies that the public "bads" (concentrations of GHGs) are assigned a positive price.

${ }^{6}$ See http://cdm.unfccc.int/Projects/registered.html. 


\section{References}

Aaheim, H. A.: 1999, 'Climate policy with multiple sources and sinks of greenhouse gases'. Environmental and Resource Economics 14(3), 413-429.

Bradford, D. F.: 2001, 'Global change: Time, money and tradeoffs'. Nature 410(6829), 649-650.

Bradford, D. F. and K. Keller: 2000, 'Global Warming Potentials: A CostEffectiveness Approach'. Mimeo, Princeton University, Cambridge, MA, USA.

Börhinger, C., A. Löschel, and T. F. Rutherford: 2005, 'Efficiency gains from "What"flexibility in climate policy: An integrated CGE assessment'. In: Venice Summer Institute: David F. Bradford Memorial Conference on The Design of Climate Policy. Venice, Italy. Selected paper.

De Cara, S., M. Houzé, and P.-A. Jayet: 2005, 'Methane and nitrous oxide emissions from agriculture in the EU: A spatial assessment of sources and abatement costs'. Environmental and Resource Economics. forthcoming.

De Cara, S. and G. Rotillon: 2003, 'Multi-greenhouse gas international agreements'. Working paper 50, INRA Economics, INRA ESR Toulouse - Grignon, France.

Eckhaus, R. S.: 1992, 'Comparing the effects of greenhouse gas emissions on global warming'. The Energy Journal 13(1), 25-35.

Fearnside, P. M.: 2002, 'Time preference in global warming calculations: A proposal for a unified index'. Ecological Economics 41(1), 21-31.

Førsund, F. R. and E. Nædval: 1998, 'Efficiency gains under exchange-rate emission trading'. Environmental and Resource Economics 12(4), 403-423.

Fuglestvedt, J. S., T. K. Berntsen, O. Godal, R. Sausen, K. P. Shine, and T. Skodvin: 2003, 'Metrics of climate change: Assessing radiative forcing and emission indices'. Climatic Change 58(3), 267-331.

Fuglestvedt, J. S., T. K. Berntsen, O. Godal, and T. Skodvin: 2000, 'Climate implications of GWP-based reductions in greenhouse gas emissions'. Geophysical Research Letters 27(3), 409-412. 
Hammit, J. K., A. Jain, J. L. Adams, and D. Wuebbles: 1996, 'A welfare-based index for assessing environmental effects of greenhouse-gas emissions'. Nature 381, 301-303.

Hayhoe, K., A. Jain, H. Keshgi, and D. Wuebbles: 2000, 'Contribution of $\mathrm{CH}_{4}$ to multi-gas reduction targets'. In: J. van Ham (ed.): Non- $\mathrm{CO}_{2}$ Greenhouse Gases: Scientific Understanding, Control and Implementation. The Netherlands, pp. 425-432, Kluwer Academic Publisher.

Hayhoe, K., A. Jain, H. Pitcher, C. MacCracken, M. Gibbs, D. Wuebbles, R. Harvey, and D. Kruger: 1999, 'Costs of multigreenhouse gas reduction targets for the USA'. Science 286, 905-906.

Hoel, M.: 1991, 'Efficient international agreements for reducing emissions of $\mathrm{CO}_{2}$ '. The Energy Journal 12, 93-107.

Houghton, J., Y. Ding, D. Griggs, M. Noguer, P. van der Linden, X. Dai, K. Maskell, and C. Johnson (eds.): 2001, Climate Change 2001: The Scientific Basis, Vol. I of IPCC Third Assessment Report. Cambridge, UK: Cambridge University Press. $881 \mathrm{p}$.

Houghton, J., G. J. Jenkins, and J. J. Ephraums (eds.): 1990, Scientific Assessment of Climate change - Report of Working Group I, Vol. I of IPCC First Assessment Report. Cambridge, UK: Cambridge University Press. 365 p.

Houghton, J., L. Meira Filho, B. Callander, N. Harris, A. Kattenberg, and K. Maskell (eds.): 1995, Climate Change 1995: The Science of Climate Change, Vol. I of IPCC Second Assessment Report. Cambridge, UK: Cambridge University Press. 572 p.

Kandlikar, M.: 1995, 'The relative role of trace gas emissions in greenhouse abatement policies'. Energy Policy 23(10), 879-883.

Kandlikar, M.: 1996, 'Indices for comparing greenhouse gas emissions: integrating science and economics'. Energy Economics 18, 265-281.

Kurosawa, A.: 2004, 'Multigas reduction strategy under climate stabilization target'. Contributed Paper 181, 7th International Conference on Greenhouse Gas Control Technologies, Vancouver, Canada. 
Manne, A. S. and R. G. Richels: 2001, 'An alternative approach to establishing trade-offs among greenhouse gases'. Nature 410, 675 - 677 .

McCarl, B. A. and U. A. Schneider: 2001, 'Greenhouse gas mitigation in U.S. agriculture and forestry'. Science 294, 2481-2482.

Mendelsohn, R., W. Morrison, M. Schlesinger, and N. Andronova: 1998, 'Countryspecific market impacts of climate change'. Climatic Change 45(3-4), 553-569.

Michaelis, P.: 1999, 'Sustainable greenhouse policies: the role of non- $\mathrm{CO}_{2}$ gases'. Structural Change and Economic Dynamics 10, 239-260.

Michaelowa, A.: 2003, 'Limiting global cooling after global warming is over - Differentiating between short- and long-lived greenhouse gases'. OPEC Review 27(4), $343-351$.

Moslener, U. and T. Requate: 2005, 'Optimal abatement in dynamic multi-pollutant problems when pollutants can be complements or substitutes'. Economics Working Paper 2005-03, Christian-Albrechts-Universitaet Kiel, Department of Economics, Kiel, Germany.

Nakicenovic, N. and R. Swart (eds.): 2000, Special Report on Emissions Scenarios. Cambridge, UK: Cambridge University Press. 612 pp.

O'Neil, B. C.: 2000, 'The jury is still out on Global Warming Potentials. An editorial comment'. Climatic Change 44(4), 427-443.

O'Neil, B. C.: 2003, 'Economics, natural science, and the costs of Global Warming Potentials. An editorial comment.'. Climatic Change 58(3), 251-260.

Ramaswamy, V., O. Boucher, J. Haigh, D. Hauglustaine, J. Haywood, G. Myhre, T. Nakajima, G. Shi, and S. Solomon: 2001, Radiative Forcing of Climate Change, Chapt. 6, pp. 349-416. Vol. I of (Houghton et al., 2001). 881 p.

Reilly, J. M., M. Babiker, and M. Mayer: 2001, 'Comparing greenhouse gases'. Global Change Report 77, MIT, Cambridge, MA, USA.

Reilly, J. M., H. D. Jacoby, and R. G. Prinn: 2003, 'Multi-gas contributors to global climate change. Climate impacts and mitigation costs of non- $\mathrm{CO}_{2}$ gases'. Report, Pew Center on Global Climate Change, Arlington, VA, USA. 60pp. 
Reilly, J. M., R. G. Prinn, J. Harnisch, J. Fitzmaurice, H. D. Jacoby, D. Kicklighter, J. Melillo, P. Stone, A. Sokolov, and C. Wang: 1999, 'Multi-gas assessment of the Kyoto Protocol'. Nature 401, 549-555.

Reilly, J. M. and K. H. Richards: 1993, 'Climate change damage and the trace gas index issue'. Environmental and Resource Economics 3, 41-61.

Sarofim, M. C., C. E. Forest, D. M. Reiner, and J. M. Reilly: 2005, 'Stabilization and global climate policy'. Global and Planetary Change. in press.

Schmalensee, R.: 1993, 'Comparing greenhouse gases for policy purposes'. The Energy Journal 14(1), 245-256.

Shine, K. P., J. S. Fuglestvedt, K. Hailemariam, and N. Stuber: 2005, 'Alternatives to the Global Warming Potential for comparing climate impacts of emissions of greenhouse gases'. Climatic Change 68(3), 281-302.

Smith, S. J. and T. M. L. Wigley: 2000, 'Global Warming Potentials: 1. Climatic implications of emissions reductions'. Climatic Change 44(4), 445-457.

Tol, R.: 2003, 'Is the uncertainty about climate change too large for expected costbenefit analysis?'. Climatic Change 56(3), 265-289.

Tol, R. S. J.: 2002a, 'Fearnside's unified index for time preference: A comment'. Ecological Economics 41(1), 33-34.

Tol, R. S. J.: 2002b, 'New estimates of the damage costs of climate change, Part II:

Dynamic estimates'. Environmental and Resource Economics 21(1), 135-160.

Tol, R. S. J., R. J. Heintz, and P. E. M. Lammers: 2003, 'Methane emission reduction: An application of FUND'. Climatic Change 57(1-2), 71-98.

UNFCCC: 1997, 'Kyoto Protocol to the United Nation Framework Convention on Climate Change'. Cop3, UNFCC (Climate Change Secretariat).

Weitzman, M. L.: 1998, 'Why the far-distant future should be discounted at its lowest possible rate'. Journal of Environmental Economics and Management 36(3), 201-208. 
